\title{
Equations différentielles p-adiques et modules de Jacquet analytiques
}

\author{
Gabriel Dospinescu *
}

November 3, 2018

\begin{abstract}
Using differential techniques, we compute the Jacquet module of the locally analytic vectors of irreducible admissible unitary representations of $\mathrm{GL}_{2}\left(\mathbf{Q}_{p}\right)$.
\end{abstract}

\section{Introduction}

Le but de cet article est d'étudier le module de Jacquet des $\mathrm{GL}_{2}\left(\mathbf{Q}_{p}\right)$ représentations de Banach unitaires admissibles, absolument irréductibles. On retrouve les résultats de [7], mais les méthodes sont sensiblement différentes.

\section{$1.1 \quad$ Notations}

On fixe une extension finie $L$ de $\mathbf{Q}_{p}$ et on note $\mathscr{R}$ l'anneau de Robba à coefficients dans $L$, i.e. l'anneau des séries de Laurent $\sum_{n \in \mathbf{Z}} a_{n} T^{n}$, avec $a_{n} \in L$, qui convergent sur une couronne de type $0<v_{p}(T) \leq r$, où $r>0$ dépend de la série.

Soit $\chi: \operatorname{Gal}\left(\overline{\mathbf{Q}_{p}} / \mathbf{Q}_{p}\right) \rightarrow \mathbf{Z}_{p}^{*}$ le caractère cyclotomique. Il induit un isomorphisme de $\Gamma=\operatorname{Gal}\left(\mathbf{Q}_{p}\left(\mu_{p^{\infty}}\right) / \mathbf{Q}_{p}\right)$ sur $\mathbf{Z}_{p}^{*}$ et on note $a \rightarrow \sigma_{a}$ son inverse. On munit $\mathscr{R}$ d'une action de $\Gamma$ et d'un Frobenius $\varphi$, en posant $\left(\sigma_{a} f\right)(T)=f\left((1+T)^{a}-1\right)$ et $(\varphi f)(T)=$ $f\left((1+T)^{p}-1\right)$. Soit $\nabla=\lim _{a \rightarrow 1} \frac{\sigma_{a}-1}{a-1}$ l'action infinitésimale de $\Gamma$. Explicitement, on a $\nabla(f)=t(1+T) f^{\prime}(T)$, où $t=\log (1+T)$.

Si $\delta: \mathbf{Q}_{p}^{*} \rightarrow L^{*}$ est un caractère continu, on note $\mathscr{R}(\delta)$ le $\mathscr{R}$-module libre de rang 1 ayant une base $e$ (dite canonique ) telle que $\varphi(e)=\delta(p) e$ et $\sigma_{a}(e)=\delta(a) e$ pour tout $a \in \mathbf{Z}_{p}^{*}$. On pose $w(\delta)=\delta^{\prime}(1)$, la dérivée de $\delta$ en 1 (rappelons que $\delta$ est automatiquement localement analytique). Si $\delta$ est unitaire (i.e. si $\delta\left(\mathbf{Q}_{p}^{*}\right) \subset O_{L}^{*}$ ), alors $w(\delta)$ est le poids de Hodge-Tate généralisé du caractère de $\operatorname{Gal}\left(\overline{\mathbf{Q}_{p}} / \mathbf{Q}_{p}\right)$ attaché à $\delta$ par la théorie locale du corps de classes, normalisée de telle sorte que $\chi$ corresponde à $x \rightarrow x \cdot|x|_{p}$.

${ }^{*}$ C.M.L.S, Ecole Polytechnique, gabriel.dospinescu@math.polytechnique.fr 


\subsection{La correspondance de Langlands locale $p$-adique}

Une $L$-représentation de $\operatorname{Gal}\left(\overline{\mathbf{Q}_{p}} / \mathbf{Q}_{p}\right)$ est un $L$-espace vectoriel de dimension finie, muni d'une action $L$-linéaire continue de $\operatorname{Gal}\left(\overline{\mathbf{Q}_{p}} / \mathbf{Q}_{p}\right)$. Les travaux de Fontaine, CherbonnierColmez, Kedlaya et Berger (voir [1, 3, 11, 12]) associent à une $L$-représentation $V$ un $\mathscr{R}$-module $D_{\text {rig }}=D_{\text {rig }}(V)$ libre de rang $\operatorname{dim}_{L} V$, muni d'actions semi-linéaires de $\varphi$ et $\Gamma$, qui commutent. $D_{\text {rig }}$ est aussi muni d'un inverse à gauche $\psi$ de $\varphi$, qui joue un grand rôle dans la théorie.

Dans la suite de cette introduction on suppose que $V$ est de dimension 2 sur $L$, absolument irréductible. La correspondance de Langlands locale $p$-adique [5] associe à $V$ un $L$-espace de Banach $\Pi=\Pi(V)$, muni d'une action continue de $\mathrm{GL}_{2}\left(\mathbf{Q}_{p}\right)$, qui en fait une représentation unitaire, admissible et topologiquement absolument irréductible. Soit $\delta_{D}=\chi^{-1} \cdot \operatorname{det} V$, que l'on voit comme caractère de $\mathbf{Q}_{p}^{*}$ et comme caractère de $\mathrm{GL}_{2}\left(\mathbf{Q}_{p}\right)$, en composant avec le déterminant. On peut utiliser les actions de $\varphi, \psi$ et $\Gamma$ pour construire un faisceau $\mathrm{GL}_{2}\left(\mathbf{Q}_{p}\right)$-équivariant sur $\mathbf{P}^{1}\left(\mathbf{Q}_{p}\right)$, dont $D_{\text {rig }}$ est l'espace des sections sur $\mathbf{Z}_{p}$. Par construction, $D_{\text {rig }}^{\psi=0}$ est l'espace des sections sur $\mathbf{Z}_{p}^{*}$ et l'application de restriction à $\mathbf{Z}_{p}^{*}$ est donnée par $\operatorname{Res}_{\mathbf{Z}_{p}^{*}}=1-\varphi \circ \psi$. Soit $w_{D}$ l'involution de $D_{\text {rig }}^{\psi=0}$ décrivant l'action de $\left(\begin{array}{ll}0 & 1 \\ 1 & 0\end{array}\right)$. L'espace des sections globales du faisceau est donc

$$
D_{\text {rig }} \otimes \mathbf{P}^{1}=\left\{\left(z_{1}, z_{2}\right) \in D_{\text {rig }} \times D_{\text {rig }} \mid \quad w_{D}\left(\operatorname{Res}_{\mathbf{Z}_{p}^{*}}\left(z_{1}\right)\right)=\operatorname{Res}_{\mathbf{Z}_{p}^{*}}\left(z_{2}\right)\right\} .
$$

On montre [5, th. V.2.20] que les vecteurs localement analytiques $\Pi^{\text {an }}$ de $\Pi$ vivent dans une suite exacte de $\mathrm{GL}_{2}\left(\mathbf{Q}_{p}\right)$-modules topologiques

$$
0 \rightarrow\left(\Pi^{\text {an }}\right)^{*} \otimes \delta_{D} \rightarrow D_{\text {rig }} \otimes \mathbf{P}^{1} \rightarrow \Pi^{\text {an }} \rightarrow 0 .
$$

\subsection{Représentations triangulines}

Dans 4, Colmez définit un espace $\mathscr{S}_{\text {irr }}$ de représentations irréductibles de dimension 2 de $\operatorname{Gal}\left(\overline{\mathbf{Q}_{p}} / \mathbf{Q}_{p}\right)$, appellées triangulines. Un point de $\mathscr{S}_{\text {irr }}$ est un triplet $s=\left(\delta_{1}, \delta_{2}, \mathscr{L}\right)$, où $\delta_{1}, \delta_{2}: \mathbf{Q}_{p}^{*} \rightarrow L^{*}$ sont des caractères continus et $\mathscr{L} \in \mathbf{P}^{1}(L)$ si $\delta_{1}=x^{k} \chi \delta_{2}(k \in \mathbf{N})$, ou $\mathscr{L} \in \mathbf{P}^{0}(L)=\{\infty\}$ si $\delta_{1} \notin\left\{x^{k} \chi \delta_{2}, k \in \mathbf{N}\right\}$. Si $s \in \mathscr{S}_{\text {irr }}$, on note $w(s)=w\left(\delta_{1}\right)-w\left(\delta_{2}\right)$, $V(s)$ la représentation associée et $D_{\text {rig }}(s)$ son $(\varphi, \Gamma)$-module sur l'anneau de Robba. On note aussi $\Pi(s)=\Pi(V(s))$. Par construction, on a une suite exacte $0 \rightarrow \mathscr{R}\left(\delta_{1}\right) \rightarrow D_{\text {rig }} \rightarrow$ $\mathscr{R}\left(\delta_{2}\right) \rightarrow 0$, dont la classe d'isomorphisme est déterminée par $\mathscr{L}$. L'espace $\mathscr{S}_{\text {irr }}$ admet une partition $\mathscr{S}_{\text {irr }}=\mathscr{S}_{*}^{\text {ng }} \amalg \mathscr{S}_{*}^{\text {cris }} \amalg \mathscr{S}_{*}^{\text {st }}$, où

- $\mathscr{S}_{*}^{\text {cris }}=\left\{s \in \mathscr{S}_{\text {irr }} \mid w(s) \in \mathbb{N}^{*}, w(s)>v_{p}\left(\delta_{1}(p)\right)\right.$ et $\left.\quad \mathscr{L}=\infty\right\}$.

- $\mathscr{S}_{*}^{\text {st }}=\left\{s \in \mathscr{S}_{\text {irr }} \mid w(s) \in \mathbb{N}^{*}, w(s)>v_{p}\left(\delta_{1}(p)\right)\right.$ et $\left.\mathscr{L} \neq \infty\right\}$.

- $\mathscr{S}_{*}^{\text {ng }}=\left\{s \in \mathscr{S}_{\text {irr }} \mid w(s) \notin \mathbb{N}^{*}\right\}$.

Supposons que $\delta_{1}$ est localement algébrique et que $V$ est une représentation irréductible de dimension 2. On démontre alors [4] que :

- $V$ correspond à un point de $\mathscr{S}_{*}^{\text {cris }}$ si et seulement si $V$ devient cristalline sur une extension abélienne de $\mathbf{Q}_{p}$;

- $V$ correspond à un point de $\mathscr{S}_{*}^{\text {st }}$ si et seulement si $V$ est une tordue par un caractère d'ordre fini d'une représentation semi-stable non cristalline. 


\subsection{Le module de Jacquet analytique}

Soit $U=\left(\begin{array}{cc}1 & \mathbf{Q}_{p} \\ 0 & 1\end{array}\right)$. Si $\pi$ est une $L$-représentation localement analytique de $\mathrm{GL}_{2}\left(\mathbf{Q}_{p}\right)$ (voir [9, 15, 16] pour les bases de la théorie), on note $J(\pi)$ son module de Jacquet naïf, quotient de $\pi$ par l'adhérence du sous-espace engendré par les vecteurs $(u-1) \cdot v$, où $u \in U$ et $v \in \pi$. Le dua 11 de $J(\pi)$ est $J^{*}(\pi)=\left(\pi^{*}\right)^{U}$ et c'est naturellement une représentation localement analytique du tore diagonal de $\mathrm{GL}_{2}\left(\mathbf{Q}_{p}\right)$. Le premier résultat est l'analogue $p$-adique d'un résultat classique de la théorie des représentations lisses, et confirme le principe selon lequel les représentations triangulines correspondent aux $\mathrm{GL}_{2}\left(\mathbf{Q}_{p}\right)$-représentations de la série principale unitaire [6].

Théorème 1.1. Soient $V$ et $\Pi$ comme dans 1.2. Alors $J^{*}\left(\Pi^{\text {an }}\right)$ est un L-espace vectoriel de dimension au plus 2 et il est non nul si et seulement si $V$ est trianguline.

Ce théorème est aussi démontré dans [7, th. 0.1], en utilisant l'action de $\varphi$ sur $D_{\text {rig }}$. Notre approche est orthogonale (elle utilise l'action infinitésimale de $\Gamma$ au lieu de celle de $\varphi$ ) et plus directe : si $u^{+}$désigne l'action infinitésimale de $U$, le résultat principal de [8] montre que le noyau de $u^{+}$sur l'espace $\left(\Pi^{\mathrm{an}}\right)^{*}$ s'identifie à l'espace des solutions de l'équation différentielle $(\nabla-a)(\nabla-b) z=0$, où $z \in D_{\text {rig }}$, $a$ et $b$ sont les poids de Hodge-Tate généralisés de $V$ et $\nabla$ est l'action infinitésimale de $\Gamma$ sur $D_{\text {rig. }}$. Cela ramène l'étude de $J^{*}\left(\Pi^{\text {an }}\right)$ à la résolution de cette équation différentielle, ce qui se fait sans mal.

Soit $\delta_{1} \otimes \delta_{2}$ le caractère $(a, d) \rightarrow \delta_{1}(a) \delta_{2}(d)$ du tore diagonal $T$ de $\mathrm{GL}_{2}\left(\mathbf{Q}_{\mathrm{p}}\right)$. Le résultat suivant précise le théorème 1.1 et correspond à [7, th. 0.6].

Théorème 1.2. Soit $s=\left(\delta_{1}, \delta_{2}, \mathscr{L}\right) \in \mathscr{S}_{\text {irr }}$.

1) Si $s \in \mathscr{S}_{*}^{\text {st }}$ ou si $w(s) \notin \mathbf{Z}^{*}$, alors $J^{*}\left(\Pi^{\text {an }}(s)\right)=\delta_{1}^{-1} \otimes \delta_{2}^{-1} \chi$.

2) Si $w(s) \in\{\ldots,-2,-1\}$, alors $J^{*}\left(\Pi^{\mathrm{an}}(s)\right)=\left(\delta_{1}^{-1} \otimes \delta_{2}^{-1} \chi\right) \oplus\left(x^{w(s)} \delta_{1}^{-1} \otimes x^{-w(s)} \delta_{2}^{-1} \chi\right)$.

3) Si $s \in \mathscr{S}_{*}^{\text {cris }}$, alors $J^{*}\left(\Pi^{\mathrm{an}}(s)\right)=\left(\delta_{1}^{-1} \otimes \delta_{2}^{-1} \chi\right) \oplus\left(x^{-w(s)} \delta_{2}^{-1} \otimes x^{w(s)} \delta_{1}^{-1} \chi\right)$ si s est non exceptionnel (i.e. si $\left.\delta_{1} \neq x^{w(s)} \delta_{2}\right)$ et $J^{*}\left(\Pi^{\mathrm{an}}(s)\right)=\left(\delta_{1}^{-1} \otimes \delta_{2}^{-1} \chi\right) \otimes\left(\begin{array}{cc}1 & v_{p}(a / d) \\ 0 & 1\end{array}\right)$ dans le cas contraire.

\subsection{L'involution $w_{D}$ et dévissage de $D_{\text {rig }} \otimes \mathbf{P}^{1}$}

On suppose que $s=\left(\delta_{1}, \delta_{2}, \mathscr{L}\right) \in \mathscr{S}_{\text {irr }}$ et on note $e_{i}$ la base canonique de $\mathscr{R}\left(\delta_{i}\right), p_{s}$ la projection canonique $D_{\text {rig }}(s) \rightarrow \mathscr{R}\left(\delta_{2}\right)$ et $\hat{e}_{2} \in D_{\text {rig }}(s)$ tel que $p_{s}\left(\hat{e}_{2}\right)=e_{2}$. Si $U$ est un ouvert compact de $\mathbf{Z}_{p}$, soit $\mathrm{LA}(U)$ l'espace des fonctions localement analytiques sur $U$ à valeurs dans $L$ et soit $\mathscr{D}(U)$ son dual topologique. Si $\delta: \mathbf{Q}_{p}^{*} \rightarrow L^{*}$ est un caracère continu, on définit une involution $w_{\delta}$ sur $\mathscr{D}\left(\mathbf{Z}_{p}^{*}\right)$ en demandant que

$$
\int_{\mathbf{Z}_{p}^{*}} \phi\left(w_{\delta} \mu\right)=\int_{\mathbf{Z}_{p}^{*}} \delta(x) \phi\left(\frac{1}{x}\right)
$$

pour tout $\phi \in \operatorname{LA}\left(\mathbf{Z}_{p}^{*}\right)$. Via l'isomorphisme $\left(\mathscr{R}^{+}\right)^{\psi=0} \simeq \mathscr{D}\left(\mathbf{Z}_{p}^{*}\right)$ donné par le théorème d'Amice, cela induit une involution $w_{\delta}$ sur $\left(\mathscr{R}^{+}\right)^{\psi=0}$, qui satisfait $w_{\delta}\left(\sigma_{a} f\right)=$

\footnotetext{
${ }^{1}$ Tous les duaux que l'on considère dans cet article sont topologiques.
} 
$\delta(a) \sigma_{\frac{1}{a}}\left(w_{\delta}(f)\right)$. Cette involution s'étend de manière unique en une involution de $\mathscr{R}^{\psi=0}$, satisfaisant la même relation que ci-dessus. Le résultat suivant fournit une description plus ou moins explicite de l'involution $w_{D}$ dans le cas triangulin. Si $s \in \mathscr{S}_{*}^{\text {cris }}$, cela permet de retrouver et renforcer le délicat lemme II.3.13 de [5].

Théorème 1.3. Pour tout $f \in \mathscr{R}^{\psi=0}$ on a $w_{D(s)}\left(f \cdot e_{1}\right)=\delta_{1}(-1) w_{\delta_{D} \cdot \delta_{1}^{-2}}(f) \cdot e_{1}$ et

$$
p_{s}\left(w_{D(s)}\left(f \cdot \varphi\left(\hat{e}_{2}\right)\right)\right)=\delta_{2}(-1) w_{\delta_{D} \cdot \delta_{2}^{-2}}(f) \cdot \varphi\left(e_{2}\right) \text {. }
$$

La très mauvaise convergence de la suit 2 définissant $w_{D}$ rend délicate une preuve directe du théorème 1.3. On déduit du théorème 1.3 le corollaire 1.4 ci-dessous qui est le point de départ [7, 14] pour l'étude des vecteurs localement analytiques de la série principale unitaire.

Soit

$$
\mathscr{R} \bigotimes_{\delta} \mathbf{P}^{1}=\left\{\left(f_{1}, f_{2}\right) \in \mathscr{R} \times \mathscr{R} \mid \quad \operatorname{Res}_{\mathbf{Z}_{p}^{*}}\left(f_{2}\right)=w_{\delta}\left(\operatorname{Res}_{\mathbf{Z}_{p}^{*}}\left(f_{1}\right)\right)\right\} .
$$

En copiant les constructions de Colmez, on munit $\mathscr{R} \otimes_{\delta} \mathbf{P}^{1}$ d'une structure naturelle de $\mathrm{GL}_{2}\left(\mathbf{Q}_{p}\right)$-module topologique (pour les détails voir [3.1). Ce module $\mathscr{R} \nabla_{\delta} \mathbf{P}^{1}$ est étroitement lié aux induites parabolique 3 , car on peut montrer qu'il vit dans une suite exacte de $\mathrm{GL}_{2}\left(\mathbf{Q}_{p}\right)$-modules topologiques

$$
0 \rightarrow\left(\operatorname{Ind}_{B}^{G}\left(\delta^{-1} \otimes 1\right)\right)^{*} \rightarrow \mathscr{R} \otimes_{\delta} \mathbf{P}^{1} \rightarrow \operatorname{Ind}_{B}^{G}\left(\chi^{-1} \delta \otimes \chi^{-1}\right) \rightarrow 0 .
$$

Corollaire 1.4. Soit $s \in \mathscr{S}_{\text {irr }}$. La suite exacte $0 \rightarrow \mathscr{R}\left(\delta_{1}\right) \rightarrow D_{\text {rig }} \rightarrow \mathscr{R}\left(\delta_{2}\right) \rightarrow 0$ induit une suite exacte de $\mathrm{GL}_{2}\left(\mathbf{Q}_{p}\right)$-modules topologiques

$$
0 \rightarrow\left(\mathscr{R} \otimes_{\delta_{D} \cdot \delta_{1}^{-2}} \mathbf{P}^{1}\right) \otimes \delta_{1} \rightarrow D_{\text {rig }} \otimes \mathbf{P}^{1} \rightarrow\left(\mathscr{R} \bigotimes_{\delta_{D} \cdot \delta_{2}^{-2}} \mathbf{P}^{1}\right) \otimes \delta_{2} \rightarrow 0
$$

Ce résultat est démontré dans [7, th. 4.6], ainsi que dans [14] (prop. 6.8) par des méthodes différentes. Il permet de donner [7, th.07] une description complète de la représentation $\Pi^{\text {an }}$ (en particulier, de montrer qu'elle est de longueur finie et d'en trouver les constituants de Jordan-Hőlder), confirmant ainsi des conjectures de Berger, Breuil et Emerton [2, 10].

\footnotetext{
${ }^{2}$ Soit $D^{\dagger}$ le sous-module surconvergent de $D_{\text {rig }}$ et $D$ le $(\varphi, \Gamma)$-module sur le corps de Fontaine $\mathscr{E}$ attaché à $V$. Il découle de la correspondance de Langlands locale $p$-adique pour $\mathrm{GL}_{2}\left(\mathbf{Q}_{p}\right)$ ([5], th. II.3.1, prop. V.2.1 et lemme V.2.4) que si $z \in D^{\dagger, \psi=0}$, la suite

$$
\sum_{i \in\left(\mathbb{Z} / p^{n} \mathbb{Z}\right)^{*}} \delta_{D}\left(i^{-1}\right)(1+T)^{i} \sigma_{-i^{2}} \varphi^{n} \psi^{n}\left((1+T)^{-i^{-1}} z\right)
$$

converge dans $D$ (mais pas dans $D^{\dagger}$ ) et sa limite $w_{D}(z)$ appartient à $D^{\dagger, \psi=0}$. L'extension de $w_{D}$ à $D_{\text {rig }}^{\psi=0}$ se fait en utilisant la densité de $D^{\dagger}$ dans $D_{\text {rig. }}$.

${ }^{3}$ On note dans la suite $\operatorname{Ind}_{B}^{G}\left(\delta_{1} \otimes \delta_{2}\right)$ l'espace des fonctions localement analytiques $f: \mathrm{GL}_{2}\left(\mathbf{Q}_{p}\right) \rightarrow L$ telles que $f\left(\left(\begin{array}{ll}a & b \\ 0 & d\end{array}\right) g\right)=\delta_{1}(a) \delta_{2}(d) f(g)$ pour tous $a, d \in \mathbf{Q}_{p}^{*}, b \in \mathbf{Q}_{p}$ et $g \in \mathrm{GL}_{2}\left(\mathbf{Q}_{p}\right)$.
} 


\subsection{Remerciements}

Ce travail est une partie de ma thèse de doctorat, réalisée sous la direction de Pierre Colmez et de Gaëtan Chenevier. Je leur suis profondément reconnaissant pour des nombreuses discussions que nous avons eues et pour leurs tout aussi nombreuses suggestions. Je voudrais aussi remercier R.Taylor, X.Caruso et L. Berger pour m'avoir invité à exposer ces résultats à l'I.A.S, dans le cadre du Workshop on Galois Representations and Automorphic Forms, et à l'E.N.S Lyon, dans le cadre de la conférence "Théorie de Hodge p-adique, équations différentielles p-adiques et leurs applications". Merci aussi à Andrea Pulita pour une discussion qui m'a permis de simplifier une démonstration et à Ramla Abdellatif, qui m'a grandement aidé à améliorer la rédaction.

\section{Le module de Jacquet de $\Pi^{\text {an }}$}

\subsection{Un résultat de finitude}

On démontre un résultat de finitude pour les équations différentielles $p$-adiques attachées aux représentations galoisiennes. Rappelons [1, V.1] que si $V$ est une $L$-représentation de $\operatorname{Gal}\left(\overline{\mathbf{Q}_{p}} / \mathbf{Q}_{p}\right)$ et si $D_{\text {rig }}$ est son $(\varphi, \Gamma)$-module sur $\mathscr{R}$, alors l'action de $\Gamma$ peut se dériver, d'où une connexion $\nabla=\lim _{a \rightarrow 1} \frac{\sigma_{a}-1}{a-1}$ sur $D_{\text {rig }}$, au-dessus de la connexion $\nabla$ sur $\mathscr{R}$ (introduite dans 1.1). Si $P \in L[X]$, notons

$$
D_{\text {rig }}^{P(\nabla)=0}=\left\{z \in D_{\text {rig }} \mid P(\nabla)(z)=0\right\} .
$$

Proposition 2.1. Soit $V$ une L-représentation quelconque de $\operatorname{Gal}\left(\overline{\mathbf{Q}_{p}} / \mathbf{Q}_{p}\right)$ et soit $D_{\text {rig }}$ son $(\varphi, \Gamma)$-module sur $\mathscr{R}$. Si $P \in L[X]$ est non nul, alors

$$
\operatorname{dim}_{L} D_{\text {rig }}^{P(\nabla)=0} \leq \operatorname{dim}_{L}(V) \cdot \operatorname{deg}(P) .
$$

Démonstration. Quitte à remplacer $L$ par une extension finie, on peut supposer que toutes les racines de $P$ dans $\overline{\mathbf{Q}_{p}}$ sont dans $L$. On démontre le théorème par récurrence sur $\operatorname{deg} P$. Pour traiter le cas $\operatorname{deg}(P)=1$, nous avons besoin de quelques préliminaires.

Lemme 2.2. On a $(\operatorname{Frac}(\mathscr{R}))^{\nabla=0}=L$.

Démonstration. Ce résultat est probablement standard, mais faute d'une référence voici une preuve. Rappelons que $\nabla(f)=t \cdot(1+T) f^{\prime}(T)$ pour $f \in \mathscr{R}$, où $t=\log (1+T)$. En particulier $\mathscr{R}^{\nabla=0}=L$. La condition $\nabla\left(\frac{f}{g}\right)=0$ équivaut à $f^{\prime} \cdot g=f \cdot g^{\prime}$. Soit $r>0$ tel que $f$ et $g$ soient analytiques sur la couronne $0<v_{p}(T) \leq r$. Si $0<r^{\prime} \leq r$ et si $z \in \mathbf{C}_{p}$ est un zéro de $g$ dans la couronne $r^{\prime} \leq v_{p}(T) \leq r$, la relation $f^{\prime} \cdot g=g^{\prime} \cdot f$ montre que $z$ a la même multiplicité (finie) dans $f$ et dans $g$. D'après des résultats standard de Lazard (voir par exemple la prop. 4.12 de [1]), le quotient $\frac{f}{g}$ est donc analytique dans la couronne $r^{\prime} \leq v_{p}(T) \leq r$. Comme cela vaut pour tout $r^{\prime} \leq r$, le quotient $\frac{f}{g}$ est analytique sur la couronne $0<v_{p}(T) \leq r$ et donc est dans $\mathscr{R}^{\nabla=0}=L$. 
Le cas $\operatorname{deg}(P)=1$ suit alors du lemme ci-dessus et du fait que le rang de $D_{\text {rig }}$ sur $\mathscr{R}$ est $\operatorname{dim}_{L}(V)$.

Lemme 2.3. Soit $\alpha \in L$. L'application naturelle $D_{\text {rig }}^{\nabla=\alpha} \otimes_{L} \mathscr{R} \rightarrow D_{\text {rig }}$ est injective.

Démonstration. Il s'agit de vérifier que si $z_{1}, z_{2}, \ldots, z_{d} \in D_{\text {rig }}^{\nabla=\alpha}$ sont libres sur $L$, alors ils sont libres sur $\mathscr{R}$. Soit (quitte à renuméroter les $z_{i}$ ) $\sum_{i=1}^{k} f_{i} \cdot z_{i}=0$ une relation de longueur minimale sur $\mathscr{R}$ et soit $g_{i}=\frac{f_{i}}{f_{1}} \in \operatorname{Frac}(\mathscr{R})$. En appliquant $\nabla$ et en utilisant le fait que $\nabla\left(z_{i}\right)=\alpha \cdot z_{i}$, on obtient $\sum_{i=1}^{k} \nabla\left(g_{i}\right) \cdot z_{i}=0$. Comme $\nabla\left(g_{1}\right)=0$, par minimalité on obtient $\nabla\left(g_{i}\right)=0$ pour tout $i$. Le lemme 2.2 permet alors de conclure.

Supposons le théorème démontré pour $\operatorname{deg} P=n$ et montrons-le pour $\operatorname{deg} P=n+1$. Soit $P=(X-\alpha) \cdot Q(X)$, avec $Q \in L[X]$ et notons $W_{1}=D_{\text {rig }}^{P(\nabla)=0}$ et $W_{2}=D_{\text {rig }}^{Q(\nabla)=0}$. Alors $\nabla-\alpha$ est un opérateur $L$-linéaire de $W_{1}$ dans $W_{2}$, dont le noyau est de dimension au plus $\operatorname{dim}_{L}(V)$ (lemme 2.3) et dont l'image est de dimension au plus $\operatorname{dim}_{L} W_{2} \leq$ $\operatorname{deg}(Q) \cdot \operatorname{dim}_{L} V$. Le résultat s'en déduit.

\subsection{Finitude et annulation du module de Jacquet}

Dans la suite on suppose que $V$ et $\Pi$ sont comme dans 1.2 (donc $V$ est de dimension 2). Pour les autres notations utilisées dans la suite (en particulier $\nabla$ et $D_{\text {rig }} \otimes \mathbf{P}^{1}$ ), voir $[1.2$ et 2.1 ,

L'ingrédient essentiel pour l'étude de $J^{*}\left(\Pi^{\mathrm{an}}\right)$ est le résultat suivant, dans lequel $u^{+}$ désigne l'action infinitésimale de l'unipotent supérieur $U$ de $\mathrm{GL}_{2}\left(\mathbf{Q}_{p}\right)$. Soient $a$ et $b$ les poids de Hodge-Tate généralisés de $V$.

Proposition 2.4. Pour tout $z=\left(z_{1}, z_{2}\right) \in D_{\text {rig }} \otimes \mathbf{P}^{1}$ on a

$$
u^{+}(z)=\left(t z_{1},-\frac{(\nabla-a)(\nabla-b) z_{2}}{t}\right) .
$$

Démonstration. On peut écrire $z=z_{1}+w \cdot\left(\varphi \circ \psi\left(z_{2}\right)\right)$, où $w=\left(\begin{array}{ll}0 & 1 \\ 1 & 0\end{array}\right)$ et où l'on voit $D_{\text {rig }}$ comme sous-espace de $D_{\text {rig }} \otimes \mathbf{P}^{1}$ comme dans 1.2. On a donc

$$
u^{+}(z)=u^{+}\left(z_{1}\right)+w \cdot u^{-}\left(\varphi\left(\psi\left(z_{2}\right)\right)\right)
$$

et le résultat découle alors de [8, th. 1].

D'après la proposition 2.1, le $L$-espace vectoriel

$$
X=\left\{z \in D_{\text {rig }} \mid(\nabla-a)(\nabla-b) z=0\right\}
$$

est de dimension au plus 4 (on fera mieux par la suite). Comme $X$ est stable par $\varphi$, on a $X \subset \varphi\left(D_{\text {rig }}\right)$, de telle sorte que $(0, z) \in D_{\text {rig }} \otimes \mathbf{P}^{1}$ pour tout $z \in X$. Rappelons 
aussi que les vecteurs localement analytiques $\Pi^{\text {an }}$ de $\Pi$ vivent dans une suite exacte de $\mathrm{GL}_{2}\left(\mathbf{Q}_{p}\right)$-modules topologiques

$$
0 \rightarrow\left(\Pi^{\mathrm{an}}\right)^{*} \otimes \delta_{D} \rightarrow D_{\text {rig }} \otimes \mathbf{P}^{1} \rightarrow \Pi^{\mathrm{an}} \rightarrow 0 .
$$

Proposition 2.5. On a une égalité de sous-L-espaces vectoriels de $D_{\text {rig }} \otimes \mathbf{P}^{1}$

$$
J^{*}\left(\Pi^{\mathrm{an}}\right) \otimes \delta_{D}=\{(0, z) \mid z \in X\} .
$$

En particulier, $J^{*}\left(\Pi^{\text {an }}\right)$ est de dimension au plus 4 sur $L$.

Démonstration. Soit $\pi=\Pi^{\text {an }}$. L'inclusion $\pi^{*} \otimes \delta_{D} \subset D_{\text {rig }} \otimes \mathbf{P}^{1}$ induit une inclusion

$$
J^{*}(\pi) \otimes \delta_{D} \subset\left(D_{\text {rig }} \otimes \mathbf{P}^{1}\right)^{U} \subset\left(D_{\text {rig }} \otimes \mathbf{P}^{1}\right)^{u^{+}=0}
$$

et, d'après la proposition 2.4, on a

$$
\left(D_{\text {rig }} \otimes \mathbf{P}^{1}\right)^{u^{+}=0}=\{(0, z) \mid z \in X\} .
$$

La proposition 2.1 montre alors que $\operatorname{dim}_{L} J^{*}(\pi) \leq 4$.

Montrons maintenant que les inclusions précédentes sont des égalités. Nous aurons besoin du résultat suivant, qui montre en particulier que $\left(D_{\text {rig }} \otimes \mathbf{P}^{1}\right)^{U}=\left(D_{\text {rig }} \otimes \mathbf{P}^{1}\right)^{u^{+}=0}$.

Lemme 2.6. Soit $M$ une L-représentation localement analytique de $\left(\begin{array}{cc}p^{\mathbf{z}} & \mathbf{Q}_{p} \\ 0 & 1\end{array}\right)$. Si $M^{u^{+}=0}$ est de dimension finie sur $L$, alors $M^{u^{+}=0}=M^{U}$.

Démonstration. Il existe $n$ tel que $M^{u^{+}=0}$ soit invariant par $\left(\begin{array}{cc}1 & p^{n} \mathbf{Z}_{p} \\ 0 & 1\end{array}\right)$. Si $m \in M^{u^{+}=0}$ et $a \in \mathbf{Q}_{p}$, on a alors pour tout $k \geq n-v_{p}(a)$

$$
\left(\begin{array}{cc}
p^{k} & 0 \\
0 & 1
\end{array}\right)\left(\begin{array}{cc}
1 & a \\
0 & 1
\end{array}\right) m=\left(\begin{array}{cc}
1 & p^{k} a \\
0 & 1
\end{array}\right)\left(\begin{array}{cc}
p^{k} & 0 \\
0 & 1
\end{array}\right) m=\left(\begin{array}{cc}
p^{k} & 0 \\
0 & 1
\end{array}\right) m,
$$

donc $\left(\begin{array}{ll}1 & a \\ 0 & 1\end{array}\right) m=m$. Cela permet de conclure.

Pour finir la preuve de la proposition 2.5, il reste à voir que $(0, z) \in \pi^{*} \otimes \delta_{D}$ pour tout $z \in X$. Les $\varphi^{n}(z)$ vivent dans $X$, qui est un $L$-espace vectoriel de dimension finie. Cela entraîne (voir [1, prop 3.2]) que $z \in \tilde{D}_{\text {rig }}^{+}$, où $\tilde{D}_{\text {rig }}^{+}=\left(\tilde{\mathbf{B}}_{\text {rig }}^{+} \otimes \mathbf{Q}_{p} V\right)^{\text {Ker } \chi}$. Mais d'après [5, lemme V.2.17], le module $\tilde{D}_{\text {rig }}^{+}$est inclus dans $\pi^{*} \otimes \delta_{D}$. Le résultat en découle (noter que $(0, z)=w \cdot z)$.

Corollaire 2.7. Si V n'est pas trianguline, alors $J^{*}\left(\Pi^{\mathrm{an}}\right)=0$.

Démonstration. Il suffit de vérifier que $X=0$ si $V$ n'est pas trianguline. Si $X \neq 0$, il existe (après avoir remplacé $L$ par une extension finie) un vecteur propre pour $\varphi$ et $\Gamma$ dans $X$. On en déduit que $V$ est trianguline (utiliser le lemme 3.2 de [4]).

\footnotetext{
${ }^{4}$ Rappelons que $\tilde{\mathbf{B}}_{\text {rig }}^{+}=\cap_{n \geq 1} \varphi^{n}\left(\mathbf{B}_{\text {cris }}^{+}\right)$.
} 


\subsection{Le module de Jacquet dans le cas triangulin}

Dans la suite on fixe un point $s=\left(\delta_{1}, \delta_{2}, \mathscr{L}\right) \in \mathscr{S}_{\text {irr }}$ et on note $V=V(s), \Pi=\Pi(s)$, etc. On note $e_{i}$ la base canonique de $\mathscr{R}\left(\delta_{i}\right)$ (rappelons que $\varphi\left(e_{i}\right)=\delta_{i}(p) e_{i}$ et $\sigma_{a}\left(e_{i}\right)=\delta_{i}(a) e_{i}$ ) et $p_{s}$ la projection de $D_{\text {rig }}$ sur $\mathscr{R}\left(\delta_{2}\right)$. Noter que les poids de Hodge-Tate généralisés de $V$ sont $w\left(\delta_{1}\right)$ et $w\left(\delta_{2}\right)$ ([4, prop 4.5]). De plus, comme $D_{\text {rig }}$ est de pente 0 et que $V$ est irréductible, la théorie des pentes de Kedlaya montre que $v_{p}\left(\delta_{1}(p)\right)=-v_{p}\left(\delta_{2}(p)\right)>0$.

Le but de cette partie est de démontrer le théorème 1.2 de l'introduction. Ce théorème découle de la proposition 2.5 et de la proposition 2.11 ci-dessous, qui détermine l'espace $X$ (défini dans 2.2). Cela va demander quelques préliminaires.

Rappelons que $s \in \mathscr{S}_{*}^{\text {cris }}$ est dit exceptionnel si $\delta_{1}=x^{w(s)} \delta_{2}$. Si $s \in \mathscr{S}_{*}^{\text {cris }}$, il existe $e_{2}^{\prime} \in D_{\text {rig }}$ tel que $p_{s}\left(e_{2}^{\prime}\right)=t^{w(s)} e_{2}$ et $\sigma_{a}\left(e_{2}^{\prime}\right)=a^{w(s)} \delta_{2}(a) e_{2}^{\prime}$ pour tout $a \in \mathbf{Z}_{p}^{*}$. Si $s$ n'est pas exceptionnel, on peut choisir $e_{2}^{\prime}$ tel que $\varphi\left(e_{2}^{\prime}\right)=p^{w(s)} \delta_{2}(p) e_{2}^{\prime}$ et alors $e_{2}^{\prime}$ est unique à multiplication par un élément de $L^{*}$ près. Si $s$ est exceptionnel, on peut choisir $e_{2}^{\prime}$ tel que $\varphi\left(e_{2}^{\prime}\right)=p^{w(s)} \delta_{2}(p) e_{2}^{\prime}+e_{1}$, et alors $e_{2}^{\prime}$ est unique à addition près d'un élément de $L e_{1}$. Ces résultats sont déduits du calcul de la cohomologie de $\mathscr{R}(\delta)$, voir [4, prop. 3.10]. On aura besoin dans la suite du résultat suivant [7, lemme 3.22] :

Lemme 2.8. Soit $V$ une L-représentation irréductible de dimension 2 de $\operatorname{Gal}\left(\overline{\mathbf{Q}_{p}} / \mathbf{Q}_{p}\right)$ et soit $D_{\text {rig }} \operatorname{son}(\varphi, \Gamma)$-module.

a) Si $D_{\text {rig }}$ possède un vecteur propre pour les actions de $\varphi$ et $\Gamma$, alors $V$ est trianguline.

b) Si $V$ correspond à un point $s \in \mathscr{S}_{*}^{\text {st }} \cup \mathscr{S}_{*}^{\text {ng }}$ ou si $s \in \mathscr{S}_{*}^{\text {cris }}$ est exceptionnel, alors les vecteurs propres pour l'action de $\varphi$ et $\Gamma$ sont dans $\cup_{k \geq 0} L^{*} \cdot t^{k} e_{1}$.

c) $S i V$ correspond à un point $s \in \mathscr{S}_{*}^{\text {cris }}$ non exceptionnel, les vecteurs propres pour $\varphi$ et $\Gamma$ sont dans $L^{*} \cdot t^{k} e_{1}$ ou dans $L^{*} \cdot t^{k} e_{2}^{\prime}$ pour un $k \geq 0$.

Le lemme suivant sera utilisé constamment dans la suite. Il fournit aussi une démonstration très directe de la proposition 1.19 de [7].

Lemme 2.9. Soit $k \in L$. L'espace des solutions de l'équation $\nabla f+k f=0$ (avec $f \in \mathscr{R}$ ) est $\{0\}$ si $k \notin\{0,-1,-2, \ldots\}$ et $L \cdot t^{-k}$ si $k \in\{0,-1,-2, \ldots\}$.

Démonstration. Soit $f \in \mathscr{R}$ une solution non nulle de l'équation $\nabla f+k f=0$ et soit $j$ le plus grand entier positif tel que $f \in t^{j} \cdot \mathscr{R}$. Posons $f=t^{j} \cdot g$, avec $g \in \mathscr{R}-t \cdot \mathscr{R}$. On a $\nabla g+(k+j) g=0$. Comme $\nabla(\mathscr{R}) \subset t \cdot \mathscr{R}$, on obtient $k+j=0$ et $g \in L$. Le résultat s'en déduit.

Notons $X_{2}=\left\{z \in D_{\text {rig }} \mid\left(\nabla-w\left(\delta_{2}\right)\right) z=0\right\}$, de telle sorte que $\left(\nabla-w\left(\delta_{1}\right)\right) X \subset X_{2}$.

Lemme 2.10. On a $X_{2}=0$ si $w(s) \notin\{0,-1,-2, \ldots\}$ et $X_{2}=L \cdot t^{-w(s)} e_{1}$ si $w(s) \in$ $\{0,-1,-2, \ldots\}$.

Démonstration. Comme $\nabla e_{j}=w\left(\delta_{j}\right) \cdot e_{j}$, l'équation $\left(\nabla-w\left(\delta_{2}\right)\right)\left(f e_{1}\right)=0$ équivaut à $\nabla f+w(s) f=0$ et l'équation $\left(\nabla-w\left(\delta_{2}\right)\right)\left(f e_{2}\right)=0$ équivaut à $\nabla f=0$ et donc à $f \in L$.

Supposons que $w(s) \notin\{0,-1, \ldots\}$. La suite exacte $0 \rightarrow \mathscr{R}\left(\delta_{1}\right) \rightarrow D_{\text {rig }} \rightarrow \mathscr{R}\left(\delta_{2}\right) \rightarrow 0$ et l'observation du premier paragraphe montrent que $X_{2}$ s'injecte dans $L e_{2}$. Supposons 
que $X_{2} \neq 0$, donc $\operatorname{dim}_{L} X_{2}=1$. Soit $x \in X_{2}-\{0\}$, donc $x \notin \mathscr{R} e_{1}$ et $x$ est vecteur propre pour $\varphi$ et $\Gamma$. Le lemme 2.8 montre que $s \in \mathscr{S}_{*}^{\text {cris }}$ n'est pas exceptionnel et qu'il existe $c \in L^{*}$ et $k \in \mathbb{N}$ tels que $x=c t^{k} \cdot e_{2}^{\prime}$. Comme $x \in X_{2}$ et $\nabla e_{2}^{\prime}=\left(w(s)+w\left(\delta_{2}\right)\right) e_{2}^{\prime}$, on obtient $w(s)=-k \in\{0,-1,-2, \ldots\}$, une contradiction. Donc $X_{2}=0$.

Supposons maintenant que $w(s) \in\{0,-1,-2, \ldots\}$. Le premier paragraphe nous fournit une suite exacte $0 \rightarrow L t^{-w(s)} e_{1} \rightarrow X_{2} \rightarrow L e_{2}$ et il reste à voir qu'elle n'est pas exacte à droite. Supposons donc qu'il existe $z \in X_{2}$ qui s'envoie sur $e_{2}$. Alors $\varphi(z)-\delta_{2}(p) z$ est dans $X_{2} \cap \mathscr{R} e_{1}=L t^{-w(s)} e_{1}$ et donc, quitte à travailler 5 avec $z+c t^{-w(s)} e_{1}$ pour un $c \in L$ bien choisi, on peut supposer que $\varphi(z)=\delta_{2}(p) z$. Soit $\gamma \in \Gamma$. Il existe $a \in L$ tel que $\gamma(z)=\delta_{2}(\chi(\gamma)) z+a t^{-w(s)} e_{1}$. Comme $\varphi$ et $\Gamma$ commutent, un petit calcul donne $a\left(p^{-w(s)} \delta_{1}(p)-\delta_{2}(p)\right)=0$, donc $a=0$. Mais alors la suite exacte $0 \rightarrow \mathscr{R}\left(\delta_{1}\right) \rightarrow D_{\text {rig }} \rightarrow \mathscr{R}\left(\delta_{2}\right) \rightarrow 0$ est scindée, contradiction.

Proposition 2.11. 1) Si $s \in \mathscr{S}_{*}^{\text {st }}$ ou si $w(s) \notin \mathbf{Z}^{*}$, alors $X=L \cdot e_{1}$.

2) Si $w(s) \in\{\ldots,-2,-1\}$, alors $X=L \cdot e_{1} \oplus L \cdot t^{-w(s)} e_{1}$.

3) Si $s \in \mathscr{S}_{*}^{\text {cris }}$, alors $X=L \cdot e_{1} \oplus L \cdot e_{2}^{\prime}$.

Démonstration. Commençons par remarquer que $e_{1} \in X$ et que $t^{-w(s)} e_{1} \in X$ (resp. $e_{2}^{\prime} \in X$ ) pour $s \in\{\ldots,-2,-1\}$ (resp. $s \in \mathscr{S}_{*}^{\text {cris }}$ ). Pour l'inclusion inverse, on va distinguer deux cas :

- Si $w(s) \notin\{0,-1,-2, \ldots\}$, la proposition précédente montre que $\left(\nabla-w\left(\delta_{1}\right)\right) z=0$ si $z \in X$, dond 6 la triangulation de $D_{\text {rig }}$ induit une suite exacte

$$
0 \rightarrow L e_{1} \rightarrow X \rightarrow\left\{f e_{2} \mid \nabla(f)=w(s) f\right\} .
$$

Si $w(s) \notin \mathbf{N}^{*}$, le lemme 2.9 montre que le terme de droite de cette suite exacte est nul et $X=L e_{1}$. Si $w(s) \in \mathbf{N}^{*}$ (ce qui inclut les cas $s \in \mathscr{S}_{*}^{\text {cris }}$ et $s \in \mathscr{S}_{*}^{\text {st }}$ ), on obtient donc (par le lemme 2.9) une suite exacte $0 \rightarrow L e_{1} \rightarrow X \rightarrow L \cdot t^{w(s)}$. Cela montre déjà que $\operatorname{dim}_{L}(X) \leq 2$ et permet de conclure dans le cas $s \in \mathscr{S}_{*}^{\text {cris }}$. Supposons que $s \in \mathscr{S}_{*}^{\text {st }}$ et montrons que cette suite n'est pas exacte à droite (et donc que $X=L \cdot e_{1}$ ). Si ce n'était pas le cas, on trouve comme dans la preuve du lemme 2.10 un $z \in X$ qui s'envoie sur $t^{w(s)} e_{2}$ et tel que $\varphi(z)=p^{w(s)} \delta_{2}(p) z$. Comme $\sigma_{a}(z)-a^{w(s)} \delta_{2}(a) z \in L \cdot e_{1}$ et comme $\varphi$ commute à $\Gamma$, on obtient facilement que $\sigma_{a}(z)=a^{w(s)} \delta_{2}(a)$, donc $z$ est propre pour $\varphi$ et $\Gamma$, ce qui contredit le lemme 2.8 .

- Si $w(s) \in\{0,-1,-2, \ldots\}$, le lemme 2.10 montre que $\left(\nabla-w\left(\delta_{1}\right)\right) X \subset X_{2} \subset \mathscr{R} e_{1}$, donc l'image de $X$ dans $\mathscr{R}\left(\delta_{2}\right)$ est contenue dans $\left\{f e_{2} \mid \nabla f-w(s) f=0\right\}$. Le dernier espace est nul si $w(s)<0$ et de dimension 1 si $w(s)=0$. Enfin,

$$
X \cap \mathscr{R} e_{1}=\left\{f e_{1} \mid \nabla(\nabla f)+w(s) \nabla f=0\right\}
$$

et ceci est de dimension 2 (resp. 1) si $w(s)<0$ (resp. $w(s)=0$ ), toujours d'après le lemme 2.9 (si $w(s)=0$, noter que la relation $\nabla(\nabla f)=0$ force $\nabla f \in L \cap t \cdot \mathscr{R}=\{0\}$ et donc $f \in L)$. Ceci permet de conclure.

\footnotetext{
${ }^{5}$ Cela utilise le fait que $p^{-w(s)} \delta_{1}(p) \neq \delta_{2}(p)$, car $v_{p}\left(\delta_{1}(p)\right)>0$ et $v_{p}\left(\delta_{2}(p)\right)<0$.

${ }^{6}$ Noter que $\left(\nabla-w\left(\delta_{1}\right)\right)\left(f e_{2}\right)=(\nabla-w(s)) f \cdot e_{2}$.
} 


\section{$3 \quad$ L'involution $w_{D}$}

On décrit l'involution $w_{D}$ sur $D_{\text {rig }}^{\psi=0}$ dans le cas où $V$ est trianguline et on démontre le théorème 1.3 .

\subsection{Le module $\mathscr{R} \otimes_{\delta} \mathbf{P}^{1}$}

Soit $\delta: \mathbf{Q}_{p}^{*} \rightarrow L^{*}$ un caractère continu. Rappelons que $\mathscr{R}^{\psi=0}$ est libre de rang 1 sur 7 $\mathscr{R}(\Gamma)$, de base $1+T$ (cela découle de [5, cor. V.1.13]). L'involution $i_{\delta}$ de $L[\Gamma]$ qui envoie $\sigma_{a}$ sur $\delta(a) \sigma_{\frac{1}{a}}$ se prolonge de manière unique en une involution $i_{\delta}$ de $\mathscr{R}(\Gamma)$ ([5, lemme V.2.3]) et on définit $w_{\delta}(f \cdot(1+T))=i_{\delta}(f) \cdot(1+T)$ si $f \in \mathscr{R}(\Gamma)$. Cela fournit une involution continue sur $\mathscr{R}^{\psi=0}$ telle que pour toute distribution $\mu$ sur $\mathbf{Z}_{p}^{*}$ on ait

$$
\begin{gathered}
w_{\delta}\left(\int_{\mathbf{Z}_{p}^{*}}(1+T)^{x} \mu\right)=w_{\delta}\left(\left(\int_{\mathbf{Z}_{p}^{*}} \sigma_{x} \mu\right) \cdot(1+T)\right)= \\
\int_{\mathbf{Z}_{p}^{*}} \delta(x) \sigma_{\frac{1}{x}} \mu \cdot(1+T)=\int_{\mathbf{Z}_{p}^{*}} \delta(x)(1+T)^{\frac{1}{x}} \mu .
\end{gathered}
$$

Donc l'involution $w_{\delta}$ que l'on vient de définir coïncide avec celle définie dans 1.5,

Soit $\operatorname{LA}\left(\mathbf{P}^{1}(\delta)\right)$ l'espace des fonctions localement analytiques $\phi: \mathbf{Q}_{p} \rightarrow L$ telles que $x \rightarrow \delta(x) \phi\left(\frac{1}{x}\right)$ se prolonge en une fonction localement analytique, muni de l'action définie par (où $g=\left(\begin{array}{ll}a & b \\ c & d\end{array}\right)$ )

$$
\left(g^{-1} \cdot \phi\right)(x)=\delta(c x+d) \phi\left(\frac{a x+b}{c x+d}\right) .
$$

Un exercice standard de la théorie des induites paraboliques identifie $\operatorname{Ind}_{B}^{G}(1, \delta) \otimes \delta^{-1}$ à $\mathrm{LA}\left(\mathbf{P}^{1}(\delta)\right)$ (en tant que $\mathrm{GL}_{2}\left(\mathbf{Q}_{p}\right)$-modules topologiques). L'application qui envoie $\mu$ sur $\left(\operatorname{Res}_{\mathbf{z}_{p}}(\mu), \operatorname{Res}_{\mathbf{z}_{p}}(w \cdot \mu)\right)$ composée avec l'isomorphisme d'Amice 8 identifie le dual de $\mathrm{LA}\left(\mathbf{P}^{1}(\delta)\right)$ (comme espace vectoriel topologique) à

$$
\mathscr{R}^{+} \bigotimes_{\delta} \mathbf{P}^{1}=\left\{\left(f_{1}, f_{2}\right) \in \mathscr{R}^{+} \times \mathscr{R}^{+} \mid \operatorname{Res}_{\mathbf{Z}_{p}^{*}}\left(f_{2}\right)=w_{\delta}\left(\operatorname{Res}_{\mathbf{Z}_{p}^{*}}\left(f_{1}\right)\right)\right\} .
$$

L'espace $\mathscr{R}^{+} \bigotimes_{\delta} \mathbf{P}^{1}$ est ainsi muni d'une action de $\mathrm{GL}_{2}\left(\mathbf{Q}_{p}\right)$. Cette action est donnée par des formules explicites comme dans [5, II.1] (voir aussi [7, 4.1]). Ces formules permettent de prolonger l'action de $\mathrm{GL}_{2}\left(\mathbf{Q}_{p}\right)$ à $\mathscr{R} \bigotimes_{\delta} \mathbf{P}^{1}$ (défini de la même manière que $\mathscr{R}^{+} \bigotimes_{\delta} \mathbf{P}^{1}$, en remplaçant $\mathscr{R}^{+}$par $\left.\mathscr{R}\right)$.

\footnotetext{
${ }^{7}$ L'anneau $\mathscr{R}(\Gamma)$ est défini par $\mathscr{R}(\Gamma)=\Lambda(\Gamma) \otimes_{\Lambda\left(\Gamma_{2}\right)} \mathscr{R}\left(\Gamma_{2}\right)$, où $\Gamma_{2}=\chi^{-1}\left(1+p^{2} \mathbf{Z}_{p}\right), \Lambda(G)$ est l'algèbre des mesures à valeurs dans $O_{L}$ sur le groupe de Lie $p$-adique $G$ et $\mathscr{R}\left(\Gamma_{2}\right)$ est défini comme l'anneau $\mathscr{R}$, en remplaçant la variable $T$ par $\gamma-1$, pour n'importe quel générateur topologique $\gamma$ de $\Gamma_{2}$.

8 Cet isomorphisme identifie $\mathscr{R}^{+}$à l'espace des distributions sur $\mathbf{Z}_{p}$ via la transformée d'Amice $\mu \rightarrow \sum_{n \geq 0} \int_{\mathbf{z}_{p}}\left(\begin{array}{l}x \\ n\end{array}\right) \mu \cdot T^{n}$
} 


\subsection{Dévissage du module $D_{\text {rig }} \otimes \mathbf{P}^{1}$}

On démontre le théorème 1.3 de l'introduction et on l'utilise ensuite pour dévisser le module $D_{\text {rig }} \otimes \mathbf{P}^{1}$. Rappelons que l'on suppose que $V$ est trianguline, correspondant à un point $s=\left(\delta_{1}, \delta_{2}, \mathscr{L}\right)$ de $\mathscr{S}_{\text {irr }}$. Rappelons aussi que $D_{\text {rig }}$ est contenu dans $D_{\text {rig }} \otimes \mathbf{P}^{1}$ (voir le paragraphe 1.2). Le résultat suivant est crucial pour la suite :

Lemme 3.1. On $a w \cdot e_{1} \in\left(D_{\text {rig }} \otimes \mathbf{P}^{1}\right)^{U}$.

Démonstration. C'est une conséquence de la proposition 2.5 et du fait que $w \cdot e_{1}=\left(0, e_{1}\right)$ et $e_{1} \in X$.

Proposition 3.2. Pour tout $f \in \mathscr{R}^{\psi=0}$ on a $w_{D}\left(f \cdot e_{1}\right)=\delta_{1}(-1) w_{\delta_{D} \delta_{1}^{-2}}(f) \cdot e_{1}$.

Démonstration. On laisse au lecteur le soin de vérifier l'identité suivante (dans laquelle $\left.w=\left(\begin{array}{ll}0 & 1 \\ 1 & 0\end{array}\right)\right)$

$$
\left(\begin{array}{ll}
1 & 1 \\
0 & 1
\end{array}\right) w \cdot\left(\begin{array}{cc}
-1 & 0 \\
0 & -1
\end{array}\right) \cdot\left(\begin{array}{cc}
-1 & 0 \\
0 & 1
\end{array}\right) \cdot\left(\begin{array}{ll}
1 & 1 \\
0 & 1
\end{array}\right) \cdot w=w \cdot\left(\begin{array}{ll}
1 & 1 \\
0 & 1
\end{array}\right) .
$$

Appliquons cette identité à $e_{1} \in D_{\text {rig }} \otimes \mathbf{P}^{1}$. Le terme de gauche est éga 99 à $\delta_{1}(-1)(1+$ $T) e_{1}$, ce qui permet donc d'écrire $w_{D}\left((1+T) e_{1}\right)=\delta_{1}(-1)(1+T) e_{1}$. Comme $\sigma_{a}\left(e_{1}\right)=$ $\delta_{1}(a) e_{1}$ et comme $w_{D}\left(\sigma_{a}(z)\right)=\delta_{D}(a) \sigma_{\frac{1}{a}}\left(w_{D}(z)\right)$, il est facile de voir que les applications $F(f)=w_{D}\left(f e_{1}\right)$ et $G(f)=\delta_{1}(-1) w_{\delta_{D} \cdot \delta_{1}^{-2}}(f) \cdot e_{1}$ sont semi-linéaires pour l'action de $i_{\delta_{D} \cdot \delta_{1}^{-1}}$. Comme elles coïncident sur $1+T$, qui est une base de $\mathscr{R}^{\psi=0}$ sur $\mathscr{R}(\Gamma)$, on obtient bien $F=G$, d'où le résultat.

Soit $\mathscr{R} e_{1} \otimes \mathbf{P}^{1}=\left(D_{\text {rig }} \otimes \mathbf{P}^{1}\right) \cap\left(\mathscr{R} e_{1} \times \mathscr{R} e_{1}\right)$.

Corollaire 3.3. $\mathscr{R} e_{1} \otimes \mathbf{P}^{1}$ est un sous-module fermé de $D_{\text {rig }} \otimes \mathbf{P}^{1}$, stable sous l'action de $\mathrm{GL}_{2}\left(\mathbf{Q}_{p}\right)$.

Démonstration. En tant qu'espace vectoriel topologique, $\mathscr{R} e_{1} \otimes \mathbf{P}^{1}$ (resp. $D_{\text {rig }} \otimes \mathbf{P}^{1}$ ) s'identifie à $\mathscr{R} e_{1} \times \mathscr{R} e_{1}$ (resp. $D_{\text {rig }} \times D_{\text {rig }}$ ). La fermeture de $\mathscr{R} e_{1} \otimes \mathbf{P}^{1}$ dans $D_{\text {rig }} \otimes \mathbf{P}^{1}$

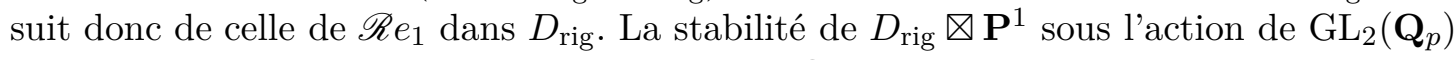
découle de la proposition 3.2, de la stabilité de $\mathscr{R} e_{1} \operatorname{par} \varphi$ et $\Gamma$, et des formules donnant l'action de $\mathrm{GL}_{2}\left(\mathbf{Q}_{p}\right)$ sur $D_{\text {rig }} \otimes \mathbf{P}^{1}$.

Proposition 3.4. Pour tout $B \in \mathscr{R}^{\psi=0}$ on a

$$
p_{s}\left(w_{D}\left(B \cdot \varphi\left(\hat{e}_{2}\right)\right)\right)=\delta_{2}(-1) w_{\delta_{D} \cdot \delta_{2}^{-2}}(B) \varphi\left(e_{2}\right) .
$$

Démonstration. Un argument de semi-linéarité comme dans la preuve de la proposition 3.2 montre que l'on peut supposer que $B=1+T$.

Posons $Y=\left(D_{\text {rig }} \otimes \mathbf{P}^{1}\right) /\left(\mathscr{R} e_{1} \otimes \mathbf{P}^{1}\right)$ et notons $z \rightarrow[z]$ la projection canonique $D_{\text {rig }} \otimes \mathbf{P}^{1} \rightarrow Y$. Nous aurons besoin du résultat suivant, analogue du lemme 3.1.

\footnotetext{
${ }^{9}$ Utiliser le fait que $w \cdot e_{1}=\left(0, e_{1}\right)$, le lemme 3.1 l'égalité $\sigma_{-1}\left(e_{1}\right)=\delta_{1}(-1) e_{1}$ et enfin le fait que $D_{\text {rig }} \otimes \mathbf{P}^{1}$ a pour caractère central $\delta_{D}$.
} 
Lemme 3.5. L'élément $\left[w \cdot \varphi\left(\hat{e}_{2}\right)\right]$ de $Y$ est invariant par $U$.

Démonstration. Le lemme 2.6 montre qu'il suffit de vérifier que $\operatorname{dim}_{L} Y^{u^{+}=0}<\infty$ et que $\left[w \cdot \varphi\left(\hat{e}_{2}\right)\right] \in Y^{u^{+}=0}$. Le $L$-espace vectoriel

$$
W=\left\{f \in \mathscr{R}\left(\delta_{2}\right) \mid\left(\nabla-w\left(\delta_{1}\right)\right)\left(\nabla-w\left(\delta_{2}\right)\right) f=0\right\}
$$

est de dimension finie d'après la proposition 2.1. Si $z=\left(z_{1}, z_{2}\right) \in D_{\text {rig }} \otimes \mathbf{P}^{1}$ satisfait $[z] \in Y^{u^{+}=0}$, alors $u^{+} z \in \mathscr{R} e_{1} \otimes \mathbf{P}^{1}$ et on déduit de la proposition 2.4 que $z_{1} \in \mathscr{R} e_{1}$ et que $p_{s}\left(z_{2}\right) \in W$. Donc

$$
z=z_{1}+w \cdot \operatorname{Res}_{p} \mathbf{Z}_{p}\left(z_{2}\right) \equiv w \cdot \operatorname{Res}_{p} \mathbf{Z}_{p}\left(p_{s}\left(z_{2}\right)\right) \quad\left(\bmod \mathscr{R} e_{1} \otimes \mathbf{P}^{1}\right),
$$

ce qui montre que $\operatorname{dim}_{L} Y^{u^{+}=0}<\infty$.

Pour conclure, il nous reste à vérifier que $u^{+}\left(w \cdot \varphi\left(\hat{e}_{2}\right)\right) \in \mathscr{R} e_{1} \otimes \mathbf{P}^{1}$. Cela découle de la proposition 2.4 et du fait que $\left(\nabla-w\left(\delta_{2}\right)\right) \hat{e}_{2} \in \mathscr{R} e_{1}\left(\operatorname{car} \sigma_{a}\left(\hat{e}_{2}\right)-\delta_{2}(a) \hat{e}_{2} \in \mathscr{R} e_{1}\right.$ pour tout $a \in \mathbf{Z}_{p}^{*}$ ).

Revenons à la preuve de la proposition 3.4. On applique l'identité matricielle du début de la preuve de la proposition 3.2 à $\left[w \cdot \varphi\left(\hat{e}_{2}\right)\right]$. Noter que $\left[w \cdot \varphi\left(\hat{e}_{2}\right)\right]$ est vecteur propre pour l'opérateur $\left(\begin{array}{cc}-1 & 0 \\ 0 & 1\end{array}\right)$, de valeur propre $\delta_{2} \cdot \delta_{D}(-1)$. L'identité matricielle s'écrit donc $p_{s}\left(w_{D}\left((1+T) \varphi\left(\hat{e}_{2}\right)\right)\right)=\delta_{2}(-1)(1+T) \varphi\left(e_{2}\right)$, ce qui permet de conclure.

Corollaire 3.6. La suite exacte $0 \rightarrow \mathscr{R} e_{1} \rightarrow D_{\text {rig }} \rightarrow \mathscr{R} e_{2} \rightarrow 0$ induit une suite exacte de $\mathrm{GL}_{2}\left(\mathbf{Q}_{p}\right)$-modules topologiques

$$
0 \rightarrow\left(\mathscr{R} \otimes_{\delta_{D} \cdot \delta_{1}^{-2}} \mathbf{P}^{1}\right) \otimes \delta_{1} \rightarrow D_{\text {rig }} \otimes \mathbf{P}^{1} \rightarrow\left(\mathscr{R} \otimes_{\delta_{D} \cdot \delta_{2}^{-2}} \mathbf{P}^{1}\right) \otimes \delta_{2} \rightarrow 0 .
$$

Démonstration. Commençons par définir les morphismes dans cette suite exacte. L'application $i$ de $\mathscr{R} \bigotimes_{\delta_{D} \cdot \delta_{1}^{-2}} \mathbf{P}^{1}$ dans $D_{\text {rig }} \otimes \mathbf{P}^{1}$ envoie $\left(f_{1}, f_{2}\right)$ sur $\left(f_{1} \cdot e_{1}, \delta_{1}(-1) f_{2} \cdot e_{1}\right)$. L'application de $D_{\text {rig }} \otimes \mathbf{P}^{1}$ dans $\mathscr{R} \bigotimes_{\delta_{D} \cdot \delta_{2}^{-2}} \mathbf{P}^{1}$ envoie $\left(A_{1} \cdot e_{1}+B_{1} \cdot \varphi\left(\hat{e}_{2}\right), A_{2} \cdot e_{1}+B_{2} \cdot \varphi\left(\hat{e}_{2}\right)\right)$ sur $\left(B_{1}, \delta_{2}(-1) B_{2}\right)$, où $A_{i}, B_{i} \in \mathscr{R}\left(\hat{e}_{2} \in D_{\text {rig }}\right.$ est un relèvement fixé de $\left.e_{2}\right)$. Le fait que ces applications $i$ et pr sont bien définies et induisent une suite exacte d'espaces vectoriels topologiques est une conséquence immédiate des propositions 3.2 et [3.4. La $\mathrm{GL}_{2}\left(\mathbf{Q}_{p}\right)$-équivariance (à torsion par $\delta_{1}$, resp. $\delta_{2}$ près) suit des propositions 3.2 et 3.4 , du fait que $f \rightarrow f \cdot e_{1}$ et $p_{s}$ sont des morphismes de $(\varphi, \Gamma)$-modules et des formules explicites donnant l'action de $\mathrm{GL}_{2}\left(\mathbf{Q}_{p}\right)$ sur les modules intervenant dans la suite exacte.

Remarque 3.7. Soit $\delta: \mathbf{Q}_{p}^{*} \rightarrow L^{*}$ un caractère continu. On a vu dans 3.1 que l'on a un isomorphisme de $\mathrm{GL}_{2}\left(\mathbf{Q}_{p}\right)$-modules topologiques

$$
\mathscr{R}^{+} \bigotimes_{\delta} \mathbf{P}^{1} \simeq \operatorname{Ind}_{B}^{G}(1, \delta)^{*} \otimes \delta \simeq\left(\operatorname{Ind}_{B}^{G}\left(\delta^{-1} \otimes 1\right)\right)^{*} .
$$


On peut vérifier (en utilisant des arguments identiques à ceux du chapitre II de [5] ; voir aussi le paragraphe 4.1 de [7]) que l'application $\mathscr{R} \otimes_{\delta} \mathbf{P}^{1} \rightarrow \operatorname{Ind}_{B}^{G}\left(\chi^{-1} \delta \otimes \chi^{-1}\right)$ définie par

$$
z \rightarrow \phi_{z}, \quad \phi_{z}(g)=\operatorname{res}_{0}\left(\operatorname{Res}_{\mathbf{z}_{p}}(w g z) \frac{d T}{1+T}\right)
$$

est une surjection $\mathrm{GL}_{2}\left(\mathbf{Q}_{p}\right)$-équivariante, de noyau $\mathscr{R}^{+} \bigotimes_{\delta} \mathbf{P}^{1}$. On dispose donc d'une suite exacte de $\mathrm{GL}_{2}\left(\mathbf{Q}_{p}\right)$-modules topologiques

$$
0 \rightarrow\left(\operatorname{Ind}_{B}^{G}\left(\delta^{-1} \otimes 1\right)\right)^{*} \rightarrow \mathscr{R} \otimes_{\delta} \mathbf{P}^{1} \rightarrow \operatorname{Ind}_{B}^{G}\left(\chi^{-1} \delta \otimes \chi^{-1}\right) \rightarrow 0 .
$$

Combiné au corollaire 3.6 et à la suite exacte

$$
0 \rightarrow\left(\Pi^{\mathrm{an}}\right)^{*} \otimes \delta_{D} \rightarrow D_{\text {rig }} \otimes \mathbf{P}^{1} \rightarrow \Pi^{\mathrm{an}} \rightarrow 0,
$$

cela permet de montrer que $\Pi^{\text {an }}$ est de longueur finie et que

$$
\left(\Pi^{\mathrm{an}}\right)^{\mathrm{ss}}=\left(\operatorname{Ind}_{B}^{G}\left(\delta_{1} \otimes \chi^{-1} \delta_{2}\right)\right)^{\mathrm{ss}} \oplus\left(\operatorname{Ind}_{B}^{G}\left(\delta_{2} \otimes \chi^{-1} \delta_{1}\right)\right)^{\mathrm{ss}} .
$$

Il faut travailler un peu plus [7] pour déterminer les extensions entre les constituants de Jordan-Hölder.

\section{Références}

[1] L.Berger-Représentations p-adiques et équations différentielles, Invent. Math. 148 (2002), p.219-284.

[2] L.Berger, C.Breuil-Sur quelques représentations potentiellement cristallines de $G_{\mathbf{Q}_{p}}$, Astérisque 330 , p. 155-211.

[3] F.Cherbonnier et P.Colmez-Représentations p-adiques surconvergentes, Invent. Math. 133 (1998), p. 581-611.

[4] P.Colmez-Représentations triangulines de dimension 2, Astérisque 319 (2008), p.213-258.

[5] P.Colmez-Représentations de $G L_{2}\left(\mathbf{Q}_{p}\right)$ et $(\varphi, \Gamma)$-modules, Astérisque 330 (2010), p. 281-509.

[6] P.Colmez-La série principale unitaire de $G L_{2}\left(\mathbf{Q}_{p}\right)$, Astérisque 330, p.213-262.

[7] P.Colmez-La série principale unitaire de $G L_{2}\left(\mathbf{Q}_{p}\right)$ : vecteurs localement analytiques, preprint.

[8] G.Dospinescu-Actions infinitésimales dans la correspondance de Langlands locale $p$-adique, à paraître à Math. Annalen.

[9] M.Emerton-Locally analytic vectors in representations of locally p-adic analytic groups, to appear in Memoirs of the AMS.

[10] M.Emerton-A local-global compatibility conjecture in the p-adic Langlands programme for $G L_{2} / \mathbb{Q}$, Pure Appl. Math. Q. 2 (2006), p. 279-393.

[11] J.-M.Fontaine-Représentations $p$-adiques des corps locaux. I, in The Grothendieck Festschrift, Vol II, Progr. Math., vol 87, Birkhauser, 1990, p. 249-309.

[12] K.S.Kedlaya-A p-adic local monodromy theorem, Ann. of Math. 160 (2004), p.93-184.

[13] R. Liu, Locally Analytic Vectors of some crystabeline representations of $\mathrm{GL}_{2}\left(\mathbf{Q}_{\mathrm{p}}\right)$, Compos. Math. (à paraître).

[14] R. Liu, B. Xie, Y. Zhang, Locally Analytic Vectors of Unitary Principal Series of $\mathrm{GL}_{2}\left(\mathbf{Q}_{\mathrm{p}}\right)$, à paraître aux Annales de l'E.N.S.

[15] P.Schneider et J.Teitelbaum- Locally analytic distributions and $p$-adic representation theory, with applications to $G L_{2}$, J. Amer. Math. Soc 15(2002), p. 443-468.

[16] P.Schneider et J.Teitelbaum- Algebras of $p$-adic distributions and admissible representations, Invent. Math. 153 (2003), p. 145-196. 